\title{
Cepheid Binaries - an Up-to-Date List
}

\author{
L. SZABADOS
}

Konkoly Observatory of the Hungarian Academy of Sciences, H-1525 Budapest, XII. P.O. Box 67, Hungary

\begin{abstract}
Cepheid variables are exceptionally useful objects for astrophysics and cosmology but at the same time these stars are normal as far as their duplicity is regarded. A brief summary is given on the relevant data of the known spectroscopicbinary Cepheids and other potential binary Cepheids, duplicity of which is suspected on the ground of various photometric methods.
\end{abstract}

\section{INTRODUCTION}

The fundamental stellar parameters (e.g., radius and mass) are usually determined with the help of either binary or intrinsically variable stars. Physical variable stars belonging to binary (or multiple) systems are even more important because the same physical property can be determined by two (or more) independent methods for these stars. In view of the role of Cepheids in the cosmological distance calibration (with the help of the period - luminosity relationship), accurate knowledge of their basic properties (especially luminosity) is essential.

\section{FREQUENCY OF BINARIES AMONG CEPHEIDS}

In fact, there is growing evidence that duplicity among Cepheids is a normal phenomenon. In order to estimate the real frequency of Cepheid binaries and the bias arising from the observational selection, duplicity status of more than 300 Cepheids brighter than $11^{m}$ was studied.

The observed distribution of binaries among Cepheids clearly indicates the following facts. The frequency of binaries exceeds $50 \%$ for the brightest (i.e. best studied) variables. The occurrence of known spectroscopic binaries continuously decreases towards fainter Cepheids, and the photometrically suspected Cepheid binaries become more important with decreasing brightness, while the spectroscopic binaries are underrepresented.

\section{THE LIST OF CEPHEID BINARIES}

In order to facilitate the selection of the objects to be studied, the relevant data on known and suspected Cepheid binaries are summarized here. The known spectroscopic-binary Cepheids are listed in Table 1, while the photometric- and visual-binary Cepheids are collected in Table 2. 
There have been several attempts to resolve Cepheids using interferometric techniques. Such data are available for eleven Cepheids (McAlister \& Hartkopf 1988). The data listed in Table 1 are useful for selecting those Cepheids which, being long-period spectroscopic binaries, can be likely separated by interferometric techniques. Cepheids listed in Table 2 are worthy of both careful radial velocity study because a considerable part of those variables may turn out to be a spectroscopic binary, and high angular resolution measurements, since the presence of a photometric or visual companion is certain.

A more detailed version of this paper will be published elsewhere.

\section{ACKNOWLEDGEMENTS}

My thanks are due to the LOC, the IAU, and the Hungarian Soros Foundation for the grants kindly offered for me. Without this financial assistance my participation in the Colloquium would not have been possible.

\section{REFERENCES}

Babel, J., Burki, G., Mayor, M., Waelkens, C., \& Chmielewski, Y. 1989, A\&A, 216, 125

Balona, L.A. 1983, Observatory, 103, 163

Burki, G. 1985, in Cepheids: Theory and Observations; Proc. IAU Coll. No. 82, ed. B.F. Madore (Cambridge: Cambridge Univ. Press) p. 34

Coulson, I.M. 1983, MNRAS, 205, 1135

Dinshaw, N., Matthews, J.M., Walker, G.A.H., \& Hill, G.M. 1989, AJ, 98, 2249

Evans, N.R. $1988, A p J S, 66,343$

Evans, N.R. 1990, PASP, 102, 551

Evans, N.R. 1992, ApJ, 384, 233

Evans, N.R., Welch, D.L., Scarfe, C.D., \& Teays, T.J. 1990, AJ, 99, 1598

Gieren, W. 1982, ApJS, 49, 1

Gieren, W.P. 1989, A\&A, 216, 135

Harris, H.C., Welch, D.L., Kraft, R.P., \& Schmidt, E.G. 1987, AJ, 94, 403

Kovács, G., Kisvarsányi, E., \& Buchler, J.-R. 1990, ApJ, 351, 606

Lloyd Evans, T. 1982, MNRAS, 199, 925

McAlister, H.A. \& Hartkopf, W.I. 1988, Second Catalog of Interferometric Measurements of Binary Stars, CHARA Contr., No. 2

Moffett, T.J. \& Barnes, T.G. III 1987, PASP, 99, 1206

Roemer, E. 1965, ApJ, 141, 1415

Slovak, M.H., Barnes, T.G. III, Evans, N.R., Welch, D.L., \& Moffett, T.J. 1989, in The Use of Pulsating stars in the Fundamental Problems of Astronomy; Proc. IAU Coll. No.111, ed. E.G. Schmidt (Cambridge: Cambridge Univ. Press), p. 284

Srabados, L. 1989, Commun. Konkoly Obs. Hung. Acad. Sci., Budapest, No. 94. (Paper I)

Ssabados, L. 1990, MNRAS, 242, 285

Szabados, L. 1991, Commun. Konkoly Obs. Hung. Acad. Sci., Budapest, No. 96. (Paper II)

Vink6, J. 1991, Ap\&SS, 183, 17

Welch, D.L., \& Evans, N.R. 1989, AJ, 97, 1153

Welch, D.L., Evans, N.R., Lyons, R.W., Harris, H.C., Barnes, T.G. III, Slovak, M.H., \& Moffett, T.J. 1987, PASP, 99, 610 
TABLE 1. Spectroscopic-binary Cepheids.

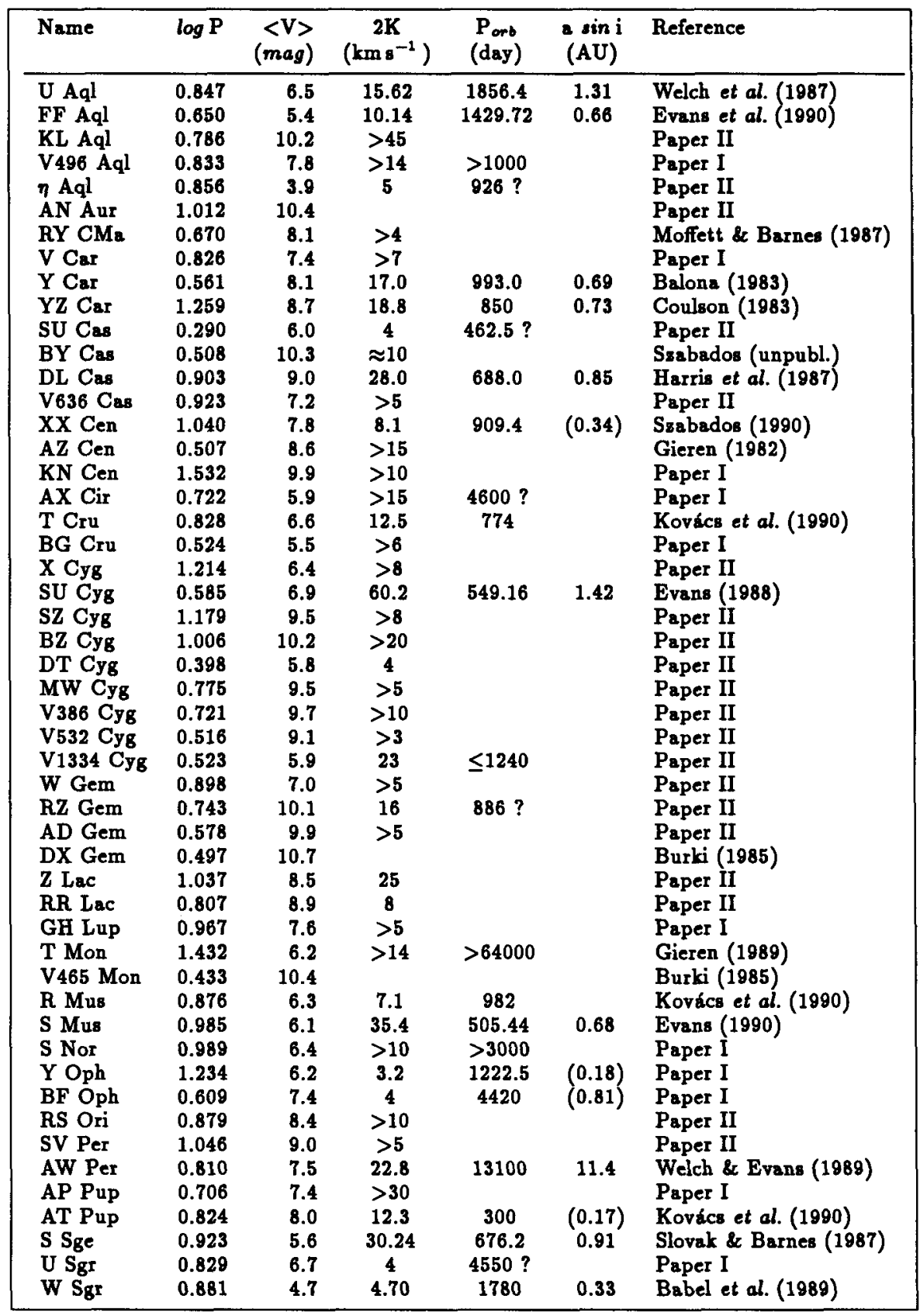


TABLE 1. Spectroscopic-binary Cepheids (continued).

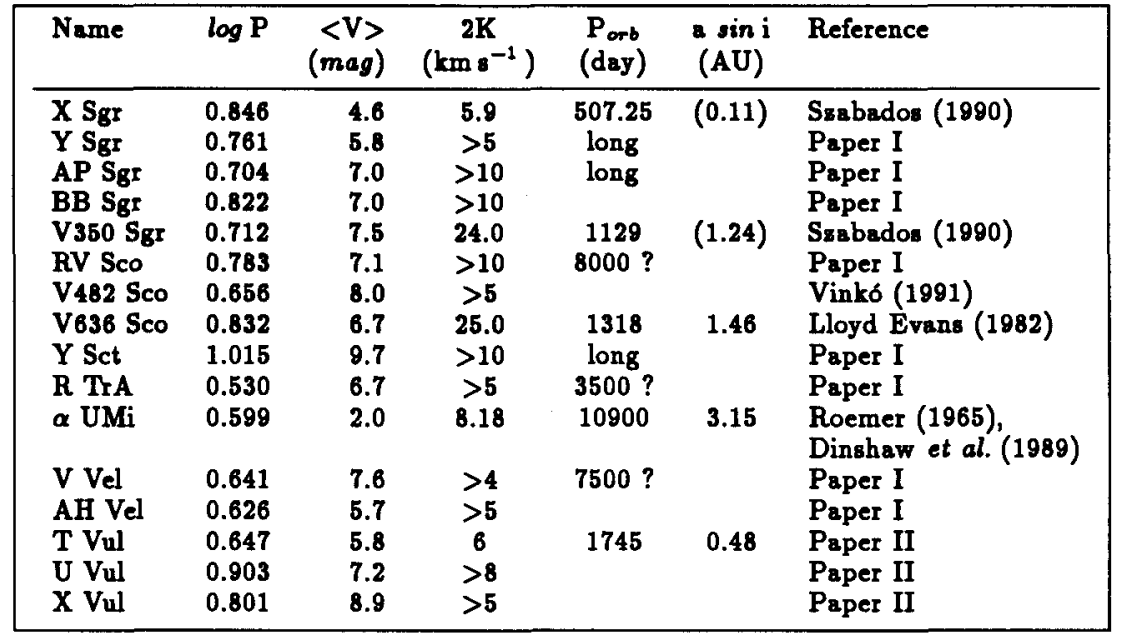

TABLE 2. Visual- and photometric-binary Cepheids*

\begin{tabular}{|c|c|c|c|c|c|c|c|}
\hline Name & $\log P$ & $\langle\mathrm{~V}\rangle$ & Feature & Name & $\log P$ & $\langle\mathrm{~V}\rangle$ & Feature \\
\hline T Ant & 0.771 & 9.3 & blue $^{1}$ & BP Cir & 0.380 & 7.5 & blue \\
\hline FN Aql & 0.977 & 8.4 & blue & SU Cru & 1.109 & 9.8 & red \\
\hline RX Aur & 1.065 & 7.7 & red $^{2}$ & AG Cru & 0.584 & 8.2 & blue \\
\hline YZ Aur & 1.260 & 10.3 & blue & VY Cyg & 0.895 & 9.6 & blue \\
\hline $\mathrm{RW}$ Cam & 1.215 & 8.7 & blue & V402 Cyg & 0.640 & 9.9 & blue \\
\hline RX Cam & 0.898 & 7.7 & blue & $\zeta \mathrm{Gem}$ & 1.006 & 3.9 & VB \\
\hline $\mathrm{RZ} \mathrm{CM}$ & 0.629 & 9.7 & blue & X Lac & 0.736 & 8.4 & blue \\
\hline WZ Car & 1.362 & 9.3 & blue & Y Lac & 0.636 & 9.2 & blue \\
\hline GI Car & 0.647 & 8.3 & blue & BG Lac & 0.727 & 8.9 & blue \\
\hline CEa Cas & 0.711 & 10.9 & $\mathrm{VB}^{3}$ & CV Mon & 0.731 & 10.3 & blue \\
\hline CEb Cas & 0.651 & 11.0 & VB & SY Nor & 1.102 & 9.5 & VB; blue \\
\hline DD Cas & 0.992 & 9.9 & blue & V440 Per & 0.879 & 6.3 & blue \\
\hline TX Cen & 1.233 & 10.5 & VB; blue & AQ Pup & 1.478 & 8.8 & blue \\
\hline UZ Cen & 0.523 & 8.8 & VB & RY Sco & 1.308 & 8.0 & VB; blue \\
\hline VW Cen & 1.177 & 10.3 & blue & V500 Sco & 0.969 & 8.8 & blue \\
\hline V659 Cen & 0.750 & 6.6 & blue & SZ Tau & 0.498 & 6.5 & blue \\
\hline \multirow{2}{*}{$\delta$ Cep } & 0.730 & 4.0 & VB & T Vel & 0.666 & 8.0 & red \\
\hline & & & & BG Vel & 0.840 & 7.7 & red \\
\hline
\end{tabular}

* Known spectroscopic binaries from Table 1 have been omitted.

1 blue companion

2 red companion

3 visual binary 